\title{
Driving and dementia: a clinician's
} guide

\author{
Sarah Wilson \& Gill Pinner
}

\begin{abstract}
SUMMARY
With an ageing population and more drivers on the road, the number of drivers with dementia is due to grow exponentially over the next 50 years. Although decisions regarding possession of a driving licence in the UK are made by the Driver and Vehicle Licensing Agency (DVLA), psychiatrists have a duty to advise patients who are unfit to drive to cease driving and to inform the DVLA of patients who pose a risk to the public by continuing to drive when advised not to. This article offers a review of the literature on dementia and driving and summarises the evidence and advice for navigating this minefield. The use of psychological test batteries in clinical practice is discussed, along with the most useful questions to ask in memory clinics. Legal guidance for various countries is considered, as is the important (but often overlooked) issue of helping older people prepare for retirement from driving.

\section{DECLARATION OF INTEREST}

G.P. is Associate Dean for Education and Training Standards at the Royal College of Psychiatrists.
\end{abstract}

Driving and dementia is a relatively new field of research. The subject first appeared in the literature in the 1980s and since then the number of studies exploring this issue has exploded. The population is ageing and the number of drivers around the world is increasing. Consequently, the increasing number of people driving with dementia will pose significant demands on the Driver and Vehicle Licensing Agency (DVLA) and on clinicians about how best to protect individuals and the wider public.

Driving is not a right. A car is a lethal weapon and drivers are granted a licence provided the strict criteria set by the DVLA are met. In the $\mathrm{UK}$, this is analogous to having a firearm or shotgun licence. In fact, the UK guidelines for driving with a mental illness (Driver and Vehicle Licensing Agency, 2012) are stricter than those for owning a gun (Thompson 2005). Driving is not safe or even possible in progressed dementias, but as awareness and diagnostic techniques improve and diagnoses are made earlier, clinicians need to be more fully informed when advising people with early dementia or mild cognitive impairment on how their driving might be affected as their condition progresses.

\section{How can clinicians assess driving risk?}

There are many difficulties in developing universal guidelines for people driving with dementia. There is no question that drivers with dementia pose a risk to themselves and other road users as their illness progresses. The challenge is assessing the level of risk and identifying at what stage the risk becomes untenable. Assessing driving ability is difficult as even in the general population there can be great variability between drivers. The symptoms of dementia can vary greatly between individuals depending on the type of dementia. This is very important when assessing driving ability.

\section{Use of self-reporting}

Studies looking at self-reports of crashes and abnormal driving behaviour have found problems with recall bias. Holland (1993) showed that most drivers perceived their own chance of having a road accident to be significantly lower, and their own skill to be greater, than that of their peers. When this is enhanced by the forgetfulness and lack of insight found in dementia, there are obvious shortcomings with using self-report alone as a method of risk assessment.

\section{Use of carer reporting}

A commonly used source of information on a patient's fitness to drive is an interview with carers, but Johnson (1998) noted that friends and relatives do not always give a true picture. This may be because they do not wish to take away the patient's independence, or because they do not wish to become burdened by a relative who can no longer drive but wishes to remain sociable. Partners may overestimate driving ability if they rely on their loved one to get around or have memory impairment themselves. Informants are also wary of being blamed for a patient's licence being taken away.

\section{ARTICLE}

Sarah Wilson is an ST5 in old age psychiatry, working in both community mental health and liaison psychiatry for older people. Her research interests include driving in dementia and the role of relatives and carers. She is currently undertaking an MSc in mental health looking at driving in dementia, supervised by Professor N. Lincoln. Gill Pinner is Director of Medical Education for Nottinghamshire Healthcare NHS Trust and Associate Dean for Education and Training Standards at the Royal College of Psychiatrists. Her research interests include aspects of disclosure of dementia diagnosis, older peoples service provision and developing communication skills training in psychiatric practice.

Correspondence Dr Sarah Wilson, Nottinghamshire Healthcare NHS Trust, Community Mental Health Team for Older People, Heather House, 72 Portland Street, Kirkby in Ashfield, Nottinghamshire NG17

7AG, UK. Email: sarahjw82@ doctors.org.uk 
Adler et al (2000) found that drivers with dementia had a greater risk of crashes and getting lost than controls, and relatives often took over responsibility for the transportation needs of older family members. They also found that concerned relatives tried to stop individuals with dementia from driving, but long-standing family dynamics often interfered with the sensitive negotiation required. Older people are more likely to accept a recommendation to stop driving from a doctor or authority figure than from a relative or friend.

Carers' reports can be unreliable, as shown in a study by Brown et al (2005). Participants (with mild, very mild or no dementia), informants and an experienced neurologist were asked to rate participants' driving ability as safe, marginal or unsafe. The participants then underwent an onroad driving assessment with a professional driving instructor. Only the neurologist's ratings were significantly related to the on-road assessment.

However, Croston et al (2009) reported that informants often observed drivers with $\mathrm{Alz}$ heimer's dementia having difficulty with traffic awareness, maintaining appropriate speeds and staying in their own lane (although in up to $25 \%$ of cases it took an on-road incident to prompt the decision to stop driving). This suggests that informants may be reliable sources of information if asked appropriate and relevant questions about driving safety.

\section{Use of other collateral information}

It may be useful to enquire whether the patient has been involved in any recent accidents, or indeed a community psychiatric nurse may report seeing dents and scratches appearing on the car. Collecting data from police records and insurance companies poses difficulties as diagnoses of dementia may be unknown, especially in the early stages, and minor bumps and offences often go unnoticed or unreported.

Two US studies (Trobe 1996; Carr 2000) have looked at state-recorded vehicle crash details and found that the crash and violation rates of individuals with dementia were not significantly different to those of age-matched controls. Both studies found, however, that patients with dementia did not drive as frequently or as far as controls, and the crash characteristics between the two groups differed, with the dementia group having more at-fault crashes.

\section{The importance of the clinical presentation}

It is really important to consider the nature of the symptom profile and natural history of the patient's specific dementia. Establishing the dementia subtype is important, as some subtypes are associated with higher risk than others. Dementia with Lewy bodies and frontotemporal dementia are the riskiest dementias in relation to driving (British Psychological Society 2001). The hallucinations associated with dementia with Lewy bodies are likely to have an impact on attention when driving, and any Parkinsonian symptoms will affect reaction times (Wood 2005). The changes in impulsivity, forward planning and shifting attention found in frontotemporal dementia mean driving skills can be affected very early in the disease process. Zuin et al (2002) found that patients with frontotemporal dementia had the highest number of accidents. They also noted that these patients had a stronger desire to continue driving and their families found it harder to try to stop them.

Studies looking at specific types of dementia have mostly focused on people with Alzheimer's disease. Most individuals with moderate to severe Alzheimer's disease are unsafe or unable to drive, so studies have focused on the mild to very mild end of the spectrum (Dubinsky 2000; Duchek 2003; Brown 2005). The preferred diagnostic tool is the Clinical Dementia Rating scale, which can distinguish between very mild and mild Alzheimer's disease (Morris 1993). This reliable and validated semi-structured interview for patients and informants takes $90 \mathrm{~min}$ to complete and gives scores of 0 (no dementia), 0.5 (very mild dementia), 1 (mild dementia), 2 (moderate dementia) and 3 (severe dementia). Very mild dementia is equivalent to mild cognitive impairment (Duchek 2003).

Drivers with a Clinical Dementia Rating score of 1 were found to have an increased risk of crashes and abnormal driving behaviours than those with scores of 0.5 (Dubinsky 2000). Overall suggestions are for individuals with mild Alzheimer's disease to have their driving status reviewed every 6 months, while those with very mild Alzheimer's disease to be reviewed every year.

The general consensus appears to be that it is usually safe to continue driving for about 3 years after the onset of Alzheimer's disease (Trobe 1996; Hopkins 2004; Breen 2007). Other dementias, such as the vascular type, may remain stable for longer, although the cognitive decline is far more unpredictable. As discussed above, frontotemporal dementia and dementia with Lewy bodies usually require individuals to stop driving much earlier. Sleep disturbance, anxiety and low mood associated with dementia may also affect driving ability by reducing concentration (Gilley 1991). 


\section{Consider comorbidities}

Older people with dementia often have multiple physical comorbidities which can affect driving ability and may require the individual to stop driving. Medications prescribed for physical comorbidities, as well as antidepressants and antipsychotics, can affect sensorimotor and perceptual abilities that may have a further detrimental effect on driving ability (Gilley 1991; Zuin 2002). The effect of cholinesterase inhibitors on driving performance has not yet been examined, but this is an important area given their increased use and benefit in milder stages of the illness.

\section{A note on the MMSE}

The Mini-Mental State Examination (MMSE; Folstein 1975) is a screening tool rather than a functional assessment and does not test functions specific to driving, i.e. executive function, judgement, divided attention and perception. Although an MMSE score of $<20 / 30$ would suggest moderate dementia and infer difficulty driving, the test is not sensitive to mild cognitive impairment and can be affected by the educational level of the patient, so risky drivers could still score $>27 / 30$ (Dubinsky 2000; Rees 2008). Although the MMSE is in common use, it is not helpful in determining whether someone with dementia is safe to drive and, indeed, its future use may be seriously curtailed owing to copyright issues.

\section{Retirement from driving}

As driving with dementia is dangerous and inevitably requires individuals to cease driving, arguments have been made that drivers with dementia have their licences revoked as soon as the diagnosis is made (Zuin 2002). However, a number of important factors need to be taken into consideration.

\section{Modification of driving behaviour}

It is important to appreciate that many older people begin to modify their driving behaviour with time, avoiding busy roads at busy times, using familiar routes and not driving in bad weather or at night. When comparing driving safety statistics and age, this needs to be taken into account. Previous perceived wisdom that older people as a group have more crashes has been disproven, as studies have established that this is due to low mileage and not increasing age (Langford 2006). Evidence suggests that patients with Alzheimer's disease usually modify their driving behaviour, but this is often not enough to reduce their crash risk completely (Adler 2000; Man-Son-Hing 2007).

\section{Impact of available alternative transport}

Around the world there are clear differences in vehicle use between rural and urban areas. Across all age groups, people are less likely to drive in cities than in the country, due to readily available public transport systems and overcrowded roads. In rural areas, a car can be a lifeline for individuals where alternative transport is non-existent and travel is required to access food and other services (Johnson 1998).

Declining mobility and physical disability associated with ageing has been shown to lead to a reliance on private vehicles, as other forms of transport such as walking and public transport are no longer realistic options (Marottoli 2000). There is also evidence that older people do not feel public transport is adequate, efficient or safe enough for their needs (Breen 2007).

\section{Driving cessation}

A number of studies have looked at driving retirement (Adler 2000; Croston 2009) and found that very few people even consider making plans for when they are no longer able to drive. This is an inevitable stage of life for most people; Foley et al (2002) showed that men tend to outlive their driving ability by 6 years and women by 10 years. Even when given a diagnosis of dementia, many individuals do not make plans for complete driving cessation (Johnson 1998; Breen 2007). This may in part be due to individuals not recognising they have impairment as a result of their cognitive state, quite different to people with physical illnesses such as epilepsy or visual problems when insight is not impaired.

Making the decision to retire from driving will have significant psychosocial consequences that may have a negative impact on people with dementia. Marottoli et al $(1997,2000)$ has shown that cessation of driving leads to a significant reduction in out-of-home activity levels, as well as an increase in depressive symptoms. It is increasingly clear that to stop driving is a lifealtering decision, reducing independence and limiting access to family, friends and services (Breen 2007).

\section{Legislation}

The legal requirements for doctors to report potentially dangerous drivers to licensing authorities vary around the world. Rosser (2000) explained how in The Netherlands, patient confidentiality takes precedence over reporting, while Danish doctors are obliged to inform the police authority if someone poses a risk. Hungary 
provides very specific guidance on psychometric testing for people with dementia wishing to acquire or retain a driving licence, while other countries still do not specifically refer to dementia in driving guidelines. In Portugal, the neurologist is the named specialist for permitting driving, and in Estonia it is the psychiatrist who makes decisions about driving with dementia.

In the USA and Canada, guidelines and obligations vary between states and provinces (AAA Foundation for Traffic Safety 2009). Physicians in all states and provinces are permitted to report medically at-risk drivers, although this is a requirement in only 16 states. One major difference between the USA and Canada and the UK and Europe is that many US and Canadian states and provinces can apply conditions or restrictions to a licence, rather than refusing to issue one at all.

\section{DVLA and the law}

In the UK, driving licences are provided by the DVLA. Since the agency was set up in 1972 , it has developed medical guidance for many illnesses that can adversely affect driving ability. Dementia has only been recognised as a specific condition in recent years. Prior to this, dementia and cognitive impairment were grouped under the generic heading of 'neurological conditions'. Over time, guidelines for many medical conditions have become clearer and tighter; however, the current guidance for drivers with dementia remains necessarily vague (Box 1).

The responsibility for renewing or revoking driving licences in the UK ultimately falls to the DVLA, but it is recognised that decisions are usually based on medical reports from doctors who care for these patients. A Canadian report by Hopkins et al (2004) noted that the responsibility for identifying drivers with dementia has fallen on the healthcare system, a role for which it was never designed or equipped to handle. They also noted that placing this responsibility on clinicians can be detrimental to the doctor-patient relationship.

Naidu \& McKeith (2006) surveyed old age psychiatrists who completed DLVA medical reports at the time and concluded that the current system for determining driving ability in people with cognitive impairment was unsatisfactory. The DVLA addressed this by developing a medical questionnaire specifically for cognitive impairment and dementia (Driver and Vehicle Licensing Agency 2009), but this still relies heavily on psychiatrists' clinical opinions, requiring yes/no answers to statements such as 'Does your patient lack insight and/or judgement to a degree that would make driving dangerous?'
BOX 1 DVLA guidance

'It is extremely difficult to assess driving ability in those with dementia. Those who have poor short-term memory, disorientation, lack of insight and judgement are almost certainly not fit to drive. The variable presentations and rates of progression are acknowledged. Disorders of attention will also cause impairment. A decision regarding fitness to drive is usually based on medical reports. In early dementia when sufficient skills are retained and progression is slow, a licence may be issued subject to annual review. A formal driving assessment may be necessary.'

(Driver and Vehicle Licensing Agency 2012: p. 35)

All drivers are required to inform the DVLA of any diagnosis or disability which may affect their driving, and it is prudent of doctors to advise patients of this. With dementia, however, a definitive diagnosis may not be made until the illness is well established, especially if the patient does not recognise the symptoms, does not have insight or is in a state of denial. Once a diagnosis is made, the same factors may still be relevant, as the patient may dispute the diagnosis or may not understand or recall the advice (British Psychological Society 2001). The General Medical Council (GMC; 2009) provide specific guidance on confidentiality and the reasons to break this if the risks and interests of public safety outweigh the interests of the patient (Box 2).

\section{Driving restrictions}

Dubinsky et al (2000) suggested that driving limitations could allow drivers with dementia to reduce their crash risk, but noted that restrictions

BOX 2 General Medical Council guidance

'If a patient continues to drive when they may not be fit to do so, you should make every reasonable effort to persuade them to stop. As long as the patient agrees, you may discuss your concerns with their relatives, friends or carers.

If you do not manage to persuade the patient to stop driving, or you discover that they are continuing to drive against your advice, you should contact the DVLA or DVA immediately and disclose any relevant medical information, in confidence, to the medical adviser.

Before contacting the DVLA you should try to inform the patient of your decision to disclose personal information. You should then also inform the patient in writing once you have done so.'

(General Medical Council 2009) 
to licences such as those suggested for young drivers may be inappropriate, as the older group's skills are expected to degenerate over time, whereas younger drivers' skills should improve with experience. Limited licences have also been rejected by Man-Son-Hing et al (2007), who suggest that drivers with dementia lack the insight to understand the rationale for the restrictions and would therefore be unlikely to adhere to them.

An alternative view was offered by Marshall $\mathcal{E}$ Gilbert (1999) who looked at physicians' attitudes and knowledge regarding assessment of fitness to drive. As previously noted, physicians felt that the need to drive was greater for rural residents than urban dwellers, and that the doctor-patient relationship was negatively affected by reporting unsafe drivers to the licensing authority. Consequently, $85.5 \%$ of physicians felt that restricted licences were a fair compromise between personal independence and public safety, and $60.3 \%$ felt that the availability of limited licences would positively influence their decision to report at-risk drivers.

\section{Driving ability tests}

\section{On-road testing}

The gold standard of fitness to drive is an on-road assessment, but this is not a readily available test. There are only 17 mobility test centres across the UK, and as well as providing driving assessments to people with dementia, they offer assessments to people of all ages with medical conditions or disabilities affecting driving skills. They also assess and advise carers who need to transport individuals with restricted mobility in their vehicles and make recommendations on vehicle modifications (see www.mobility-centres.org.uk).

Clearly, the centres do not have the capacity to offer even single tests to everyone with dementia, let alone repeated tests as the condition deteriorates. Even if on-road tests were readily available, the cost of repeated tests would be untenable, requiring reams of paperwork, which would be difficult to coordinate with 6-monthly follow-ups in memory clinics scattered around the region.

Regular testing may also be detrimental to the patient with early dementia, as the added stress and anxiety of getting to the centre (which could be up to 50 miles away) and being tested, could lead to false failures. For this reason, much research has focused on finding a simple test or battery of tests which can be carried out in memory clinics.

\section{In-office testing}

Researchers have been trying to develop batteries of so-called 'in-office tests' to better evaluate driving safety and ability in dementia, bringing together more specific psychometrics and effective probing collateral reports.

Molnar et al (2006) completed a systematic review of simple in-office cognitive tests which claimed to differentiate drivers with dementia as 'safe' or 'unsafe'. They found that commonly recommended tests such as the MMSE or Trail Making Test B did not demonstrate robustly positive findings across studies and that the Clock Drawing Test was not evaluated in any study. Cutoff scores were only reported in one study, making any meaningful comparison of studies impossible and preventing the creation of evidence-based guidelines to allow clinicians to identify patients as safe or unsafe to drive. Molnar et al explored this further and suggested alternative solutions. The most commonly employed technique currently is stratification, where rather than a dichotomous pass/fail outcome, there is a third 'uncertain' or 'indeterminate' outcome, which can suggest the need for further assessment such as the on-road driving test.

\section{Current tests that have been developed}

The USA and Canada

Office-based tests in the USA and Canada include the SAFE DRIVE checklist (Wiseman 1996), the Canadian Driving Research Initiative for Vehicular Safety in the Elderly (CanDRIVE) assessment algorithm (Man-Son-Hing 2004) and the Ottawa Driving and Dementia Toolkit (Champlain Dementia Network, Regional Geriatric Program of Eastern Ontario 2009). The SAFE DRIVE and CanDRIVE are prompts to ask questions about driving (Boxes 3 and 4).

The Ottawa Driving and Dementia Toolkit is a whole assessment package which starts with a 10-minute checklist to ensure all areas of potential concern are considered. Information is collected on the type of dementia and the functional impact of the illness along with a medical and medication

\section{BOX 3 SAFE DRIVE checklist}

Safety record

Attention skills

Family report

Ethanol use

Drug use

Reaction time

Intellectual impairment

Vision and visuospatial function

Executive functions 
BOX 4 CanDRIVE assessment acronym

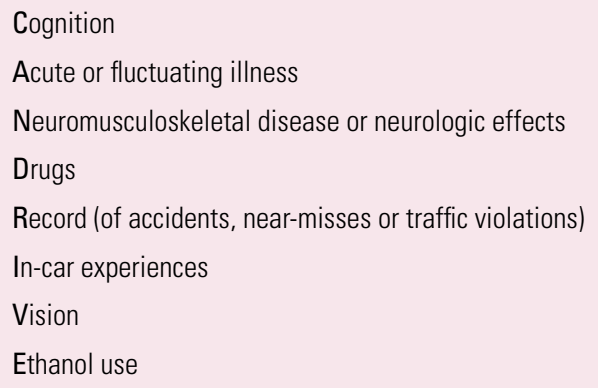

history, tests of vision, executive functioning, reaction times, insight and any family concerns (Box 5). The results of each area are then fed into an assessment algorithm.

The toolkit identifies the most pertinent question for relatives in 'the granddaughter question': 'Would you feel it was safe if a 5-year-old granddaughter was in the car alone with the person driving?' Relatives who may previously have described the patient as a safe driver often feel that the above situation would be unsafe and this is generally a sensitive indication of an unsafe driver (Molnar 2005).

The algorithm offers three potential outcomes:

1 the patient may be deemed safe to continue driving and be reassessed in 6 months

2 the patient may be clearly unsafe and need to stop driving immediately

3 the patient may lie somewhere in the middle requiring further assessment.

The toolkit contains resources to support the clinician in telling a patient they need to stop driving, ensuring documentation is completed effectively and helping all patients plan for driving retirement. As the only comprehensive toolkit of its kind, it represents a useful model of an off-road, in-office driving assessment.

\section{The UK}

In the UK, research has been more dispersed. Government-funded initiatives recognise the need for clearer guidance on driving and dementia and better systems than we currently have (British Psychological Society 2001). Psychological batteries developed for neurological conditions such as multiple sclerosis and stroke have been tested on people with dementia and adapted to provide test batteries which can be used to assess driving ability. The two major test batteries of this nature are the Dementia Drivers Screening Assessment developed by Lincoln et al (2006) from the earlier Stroke Drivers Screening Assessment, and the Rookwood Driving Battery developed by McKenna et al (2004) for anyone with acquired brain pathology.

Although these two tests may be too long and cumbersome to complete in a 30-minute memory clinic appointment alongside medication reviews and issues raised by the patient and their relatives (both tests take about 30 min to administer), they may be manageable in 6-monthly appointments with a clinical psychologist, if this resource is available. This is likely to be more acceptable to the patient and more cost-effective than referring every driver with dementia for a regular on-road assessment.

Dementia Drivers Screening Assessment (DDSA) The DDSA was published in 2006, following work by Radford in 2001. It looked at nine cognitive assessments covering a range of cognitive domains likely to affect driving ability, such as attention, memory, executive functioning, reasoning and visuospatial skills. When validated against an on-road driving test, no single test was able to differentiate between safe and unsafe drivers, consistent with the finding of Molnar et al (2006). Further analysis identified a combination of six tests which correctly classified $92 \%$ of drivers with dementia as safe or unsafe.

Lincoln et al (2006) noted that ex-professional drivers were likely to be identified as unsafe on the cognitive assessments, but safe on the road, whereas patients with spatial problems performed

BOX 5 Ottowa Driving and Dementia Toolkit 10-minute checklist

- Dementia type (dementia with Lewy bodies and frontotemporal dementia most unsafe)

- Functional impact (personal and instrumental activities of daily living)

- Family concerns (ask separate from the patient, 'the granddaughter question')

- Visuospatial (intersecting pentagons/Clock Drawing Test)

- Physical ability to operate a car (neuromusculoskeletal and cardiac history)

- Vision/visual fields

- Drugs (medication, alcohol, drowsiness or slowed reaction time)

- Trail Making Test A \& B

- Ruler Drop Reaction Time Test

- Judgement/insight (e.g. 'What would you do if you were driving and saw a ball roll out on the street ahead of you?', 'With your diagnosis of dementia, do you think at some time you will need to stop driving?') 
poorly on the on-road test, but were not identified by the cognitive assessments. A further study by Lincoln et al (2009) looked at the accuracy of the DDSA. The authors found that the assessment battery correctly classified $76.2 \%$ of participants as safe or unsafe to drive, and recommended that it be used to stratify patients into safe, unsafe and a third 'uncertain' group who might benefit from further testing such as an on-road assessment.

Rookwood Driving Battery (RDB) The RDB was proposed in 1998 by McKenna, who noted that traditional psychometric tests used to assess fitness to drive were often IQ-related and bore little relation to the cognitive systems specifically involved in driving behaviour. McKenna aimed to construct a flexible test battery incorporating specific tests for 'each identifiable function underlying movement and analysis of the visual world as they relate to driving' and 'a method of measuring the integrity of cognitive functioning as it relates to driving?

The RDB was launched in 2004 (McKenna 2004) and it comprised a battery of 12 tests. In a comparison with an on-road driving test the RDB had $92 \%$ accuracy in predicting those with brain injury or pathology who would fail the on-road test and a $91 \%$ predictive accuracy of those who would pass. The test was much less accurate for those aged 70 and above, with only $85 \%$ accuracy for predicting an on-road fail and $37 \%$ for a pass.

A further study (Rees 2008) collected normative older adult data to enable more appropriate use of the battery with older participants. Significant differences were found in performance on the RDB for people over 70 years suggesting that the pass/ fail scoring system developed for younger adults would give cognitively intact older adults a higher baseline error score. Rees and colleagues suggested several cut-offs for pass/fail, but proposed that a clear fail on the battery still predicts a high fail rate on the road, making the RDB a useful screening tool. As with the DDSA, the RDB allows for an indeterminate outcome requiring further assessment.

\section{Conclusions}

The key points of this article are summarised in Box 6. Doctors in the UK should inform drivers with dementia to advise the DVLA of their diagnosis. Not all patients with this condition will follow this advice and if a patient is deemed unsafe to drive but continues to do so, the GMC (2009) provides guidance on contacting the DVLA without the patient's consent. It is important to differentiate diagnostic subtypes as specific cognitive deficits are more likely to be associated
B0X 6 Key points

- Decisions regarding possession of a driving licence are ultimately made by the DVLA

- Driving is usually safe for 3 years after the onset of Alzheimer's disease

- The risks are much greater with dementia with Lewy bodies and frontotemporal dementia

- Early discussion of driving retirement is imperative in helping older people prepare for cessation

- Older people are more likely to accept a recommendation to stop driving from a doctor or authority figure than from a relative or friend

- In-office tests offer three outcomes: pass, fail or need for further assessment

- An on-road driving test is the gold standard assessment, but availability is limited

with increased risk. Patients with frontotemporal dementia or dementia with Lewy bodies pose the greatest risk. Cognitive assessments, algorithms and pertinent questions are available to categorise patients as safe, unsafe or needing further assessment. The gold standard assessment is an on-road driving test, where this as available. It is advisable to discuss driving cessation as early as possible with all elderly patients, to help them prepare for the future.

\section{Acknowledgements}

Many thanks to Professor Nadina Lincoln at Nottingham University for her advice, support and supervision.

\section{References}

AAA Foundation for Traffic Safety (2009) Driving Licensing Policies and Practices database. AAAFTS (http://lpp.seniordrivers.org/lpp/index.cfm).

Adler G, Rottunda S, Bauer M, et al (2000) Driving cessation and Alzheimer's dementia: Issues confronting patients and family. American Journal of Alzheimer's Disease and Other Dementias 15: 212-6.

Breen DA, Breen DP, Moore JW, et al (2007) Driving and dementia. BMJ 334: 1365-9.

British Psychological Society (2001) Fitness to Drive and Cognition: A Document of the Multi-Disciplinary Working Party on Acquired Neuropsychological Deficits and Fitness to Drive 1999. BPS.

Brown L, Ott B, Papandonatos D, et al (2005) Prediction of on-road driving performance in patients with early Alzheimer's disease. Journal of the American Geriatrics Society 53: 94-8.

Carr D, Duchek J, Morris J (2000) Characteristics of motor vehicle crashes of drivers with dementia of the Alzheimer type. Journal of the American Geriatrics Society 48: 18-22.

Champlain Dementia Network, Regional Geriatric Program of Eastern Ontario (2009) The Driving and Dementia Toolkit for Health Professionals (3rd edn). CDN, RGPEO (http://www.champlaindementianetwork.org/ uploads/Resources/kitjune09.pdf).

Croston J, Meuser T, Berg-Weger M, et al (2009) Driving retirement in older adults with dementia. Topics in Geriatric Rehabilitation 25: 154-62.
MCO answers

$1 \mathrm{c} \quad 2$ b 3 e 4 b $5 d$ 
Dubinsky R, Stein A, Lyons K (2000) Practice parameter: risk of driving and Alzheimer's disease (an evidence-based review). Report of the Quality Standards Subcommittee of the American Academy of Neurology. Neurology 54: 2205-11.

Duchek J, Carr D, Hunt L, et al (2003) Longitudinal driving performance in early-stage dementia of the Alzheimer's type. Journal of the American Geriatrics Society 51: 1342-7.

Driver and Vehicle Licensing Agency (2009) Questionnaire to Assess Your Patient's Medical Fitness to Drive (CG2C). Available from DVLA, Swansea.

Driver and Vehicle Licensing Agency (2012) For Medical Practitioners. At A Glance Guide to the Current Medical Standards of Fitness to Drive. DVLA

Foley D, Heimovitz H, Guralnik J, et al (2002) Driving life expectancy of persons aged 70 years and older in the United States. American Journal of Public Health 92: 1284-9.

Folstein MF, Folstein SE, McHugh PR (1975) 'Mini mental state'. A practical method for grading the cognitive state of patients for the clinician. Journal of Psychiatric Research 12: 189-98.

Gilley D, Wilson R, Bennett D, et al (1991) Cessation of driving and unsafe motor vehicle operation by dementia patients. Archives of Internal Medicine 151: 941-6.

General Medical Council (2009) Confidentiality: Reporting Concerns about Patients to the DVLA or the DVA. GMC

Holland C (1993) Self-bias in older drivers' judgments of accident likelihood. Accident Analysis and Prevention 25: 431-41.

Hopkins R, Kilik L, Day D, et al (2004) Driving and dementia in Ontario: a quantitative assessment of the problem. Canadian Journal of Psychiatry 49: 434-8

Johnson J (1998) Older rural adults and the decision to stop driving: the influence of family and friends. Journal of Community Health Nursing 15: $205-16$

Langford J, Methorst R, Hakamies-Blomqvist L (2006) Older drivers do not have a high crash risk - a replication of low mileage bias. Accident Analysis and Prevention 38: 574-8.

Lincoln N, Radford K, Lee E, et al (2006) The assessment of fitness to drive in people with dementia. International Journal of Geriatric Psychiatry 21: 1044-51.

Lincoln NB, Taylor JL, Vella K, et al (2009) A prospective study of cognitive tests to predict performance on a standardised road test in people with dementia. International Journal of Geriatric Psychiatry 25: 489-96.

McKenna P (1998) Fitness to drive: a neuropsychological perspective. Journal of Mental Health 7: 9-18.

McKenna P, Jefferies L, Dobson A, et al (2004) The use of a cognitive battery to predict who will fail an on-road driving test. British Journal of Clinical Psychology 43: 325-36.
Man-Son-Hing M, Marshall S, Molnar F, et al (2004) A Canadian research strategy for older drivers: the CanDRIVE Program. Geriatrics Today: Journal of the Canadian Geriatric Society 7: 86-92.

Man-Son-Hing M, Marshall S, Molnar F, et al (2007) Systematic review of driving risk and the efficacy of compensatory strategies in persons with dementia. Journal of the American Geriatrics Society 55: 878-84.

Marottoli R, Mendes de Leon C, Glass T, et al (1997) Driving cessation and increased depressive symptoms: prospective evidence from the New Haven EPESE. Journal of the American Geriatrics Society 45: 202-6.

Marottoli R, Mendes de Leon C, Glass T, et al (2000) Consequences of driving cessation: decreased out-of-home activity levels. Journal of Gerontology: Psychological Sciences 55B: S334-40.

Marshall S, Gilbert N (1999) Saskatchewan physicians' attitudes and knowledge regarding assessment of medical fitness to drive. Canadian Medical Association Journal 160: 1701-4.

Molnar F, Byszewski A, Marshall S, et al (2005) In-office evaluation of medical fitness to drive: practical approaches for assessing older people. Canadian Family Physician 51: 372-9.

Molnar F, Patel A, Marshall S, et al (2006) Clinical utility of office-based cognitive predictors of fitness to drive in persons with dementia: a systematic review. Journal of the American Geriatrics Society 54: 1809-24.

Morris J (1993) The Clinical Dementia Rating (CDR): current version and scoring rules. Neurology 43: 2412-4.

Naidu A, McKeith IG (2006) Driving, dementia and the Driver and Vehicle Licensing Agency: a survey of old age psychiatrists. Psychiatric Bulletin 30: $265-8$

Radford K (2001) Validation of the Stroke Drivers Screening Assessment for Patients with an Acquired Neurological Disability (PhD thesis). University of Nottingham.

Rees J, McKenna P, Bell V, et al (2008) The Rookwood Driving Battery: normative older adult performance. British Journal of Clinical Psychology 47: 139-51.

Rosser M (2000) Dementia and driving: European National Guidelines. European Journal of Neurology 7: 745

Thompson P (2005) Psychiatric illness and gun licensing. Psychiatric Bulletin 29: 281-3.

Trobe J, Waller P, Cook-Flannagan C, et al (1996) Crashes and violations among drivers with Alzheimer disease. Archives of Neurology 53: 411-6.

Wiseman E, Souder E (1996) The older driver: a handy tool to assess competence behind the wheel. Geriatrics 51: 36-45.

Wood J, Worringham C, Kerr G, et al (2005) Quantitative assessment of driving performance in Parkinson's disease. Journal of Neurology, Neurosurgery and Psychiatry 76: 176-80.

Zuin D, Ortiz H, Boromei D, et al (2002) Motor vehicle crashes and abnormal driving behaviours in patients with dementia in Mendoza, Argentina. European Journal of Neurology 9: 29-34.

\section{MCOs}

Select the single best option for each question stem

1 The type of dementia perceived to pose the highest risk when driving is:

a vascular dementia

b Alzheimer's disease

c dementia with Lewy bodies

$\mathrm{d}$ mild cognitive impairmen

e dementia with mixed aetiology.

2 The most sensitive and pertinent question for relatives or carers is:

a What make/model of car does the patient drive?

b Would you feel it was safe if a 5-year-old child was in the car alone with the person driving? c Do you feel safe in the car with the person driving?

$\mathrm{d}$ Has the person had any speeding tickets or other traffic violations in the past 2 years?

e Has the person ever gone through a red light?

3 Which of the following is not identified by the DVLA as deeming a patient almost certainly not fit to drive:

a poor short-term memory

b disorientation

c lack of insight

d lack of judgement

e MMSE score of less than 20/30
4 Both the DDSA and the RDB take how long to administer:

a $20 \mathrm{~min}$

b $30 \mathrm{~min}$

c $45 \mathrm{~min}$

d $60 \mathrm{~min}$

e $90 \mathrm{~min}$.

5 How many years do women tend to outlive their driving ability by:

a 4

b 6

c 8

d 10

e 20 